\title{
Restoration of Worn Parts of Peat Digging Machines by Plastic Deformation
}

\author{
Igor Gorlov ${ }^{1, *}$, Alexander Yablonev ${ }^{1}$, Larisa Kozyreva ${ }^{1}$, and Konstantin Fomin ${ }^{1}$ \\ ${ }^{1}$ Tver State Technical University (TvSTU), 170026, Afanasy Nikitin emb., 22, Tver, Russia
}

\begin{abstract}
Reduction in production and phase-out of a significant range of peat machines leads to a lack of spare parts for the equipment on the balance sheet of peat enterprises. Partial compensation for a shortcoming of spare parts is possible due to the restoration of worn parts. The article describes a new method of restoring a worn surface by plastic deformation, which implements the effect of increased plasticity of a material. The effect ensures the qualitative repair of friction units made of plastic varieties. The proposed restoring method allows obtaining the necessary surface parameters without complex technological equipment and provides improvement of mechanical characteristics and acquisition of new tribotechnical properties. There are the results of complex studies of the deformation process under superimposed hydrostatic pressure.
\end{abstract}

\section{Introduction}

The increase in peat harvesting in a number of countries and revival of the peat industry in Russia are due to the fact that there is a steady trend of deterioration in geological conditions of oil and gas production. According to 2009 development plan, the EU established an energy program called "20: 20: 20: 20", which requires a $20 \%$ reduction in electricity consumption, a $20 \%$ increase in the part of renewable energy sources in total consumption and a $20 \%$ reduction in greenhouse gas emission by 2020 [1]. The use of peat as a renewable energy source will help to solve these problems partially, provided that the efficiency of its harvesting is increased [2].

In Russia, the lack of sufficient production capacity in peat engineering has escalated the problem of restoring the peat industry. Due to the collapse in peat harvesting in the 2000 s, the purchase of peat equipment was drastically reduced, which led to almost complete halt of peat engineering in our country. As a result, factories that manufacture peat equipment were forced to reorganize their production within a very short time to produce technological machines for other purposes. This led to the fact that former specialized plants has begun to produce peat equipment in a single quantity on a contractual basis. Thus, its cost was forced up and the quality decreased. Due to such situation in the field of making peat equipment, peat enterprises were forced to purchase imported equipment, so the production of peat machines in Russia almost completely ceased. Another problem that arose due to the phase-out of peat machines in our country is the lack

\footnotetext{
${ }^{*}$ Corresponding author: gorloviv@yandex.ru
} 
of quality spare parts for repair. Organizations that repair peat equipment have to produce the necessary parts themselves as a single production, which increases their cost and does not provide the required quality. An alternative to such production is restoring worn parts by modern methods or increasing the life of parts by applying new lubrication systems in friction units [3].

The production of the restored parts is actively developing all over the world. Great attention is payed to the problems of improving the quality of operation and repair $[4,5]$. There are new approaches in restoration of worn parts, which provide an increased resource for repaired units. One of the methods is restoration of locally worn parts by plastic deformation along a conductor surface (PDCS). Various methods can be used to assess the quality of the recovered friction surfaces [6].

\section{Materials and Methods}

Traditional restoration methods use plastic deformation, which requires additional machining after deformation, leading to material losses. In contrast, the proposed PDCS method allows obtaining the required parameters of a reconstructed surface without using complex technological equipment, while ensuring the improvement of mechanical characteristics and acquisition of qualitatively new tribotechnical properties of support surfaces. In addition, low cost and technological simplicity of the proposed recovery method is also important.

The reconstructed surface in general is formed due to combining local parts obtained after deformation at the established points of the worn out part. Fig. 1 shows a layout diagram of the device for recovery.

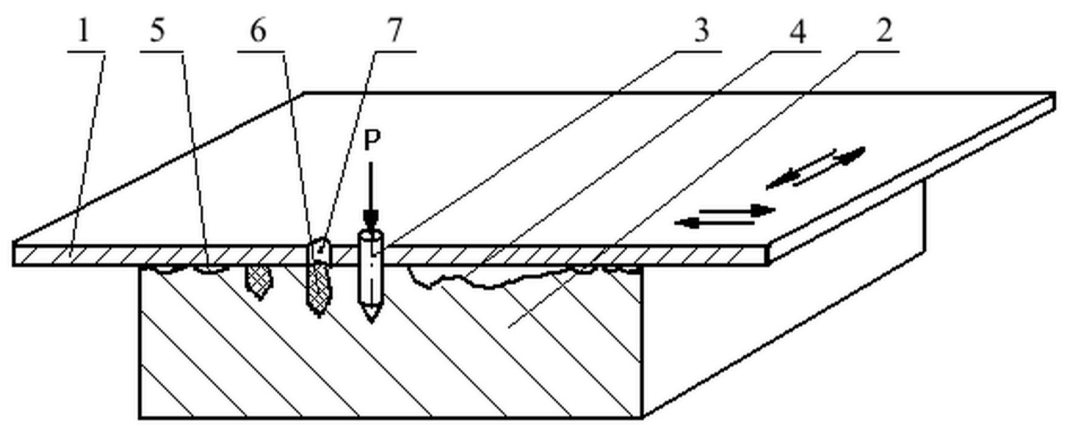

Fig. 1. A recovery method for a locally worn surface. Patent RU №2447981 C2

The method is implemented as follows. A buckle arrestor 1 (a conductor with a nominal surface shape) is placed on the part 2 so that its adjoining surface corresponds to the position of the nominal surface contour. A topogram (three-dimensional model) of the worn out part 4 of the surface is taken in predetermined areas using the indenter 3.

The topogram helps to determine points and values of plastic deformation (sections 5 do not require restoration). Step by step using $\mathrm{P}$ and the indenter 3 in the worn part the recesses 6 are obtained by plastic deformation. Due to these recesses the areas of the worn surface 4 rise up to the stop in a conductor and form a restored surface of the nominal profile, which does not require further machining. After completing all stages of plastic deformation, the recesses 6 are filled through the hole 7 with antifriction material and the limiter 1 is removed from the restored part 2. As a result, there is a reconstructed surface with new tribotechnical properties.

PDCS makes it possible to restore a nominal surface of a worn sliding bearing with a different wear rate [7]. The wear distribution along the working surface is local. Therefore, 
the effect on the deformable material should be related to the wear rate at each particular point. The restoration of the entire worn surface consists of separate surfaces obtained when introducing an indenter at the corresponding section.

The methods of statistical mechanics can be used [8] to analyze the process of plastic deformation. However, the difficulties related to the implementation of such methods in PDCS conditions can lead to ambiguous results. Computer modeling is also helpful $[9,10]$, but the change of plastic properties of the recovered material under PDCS conditions cannot be taken into account now in the corresponding information analysis systems. Mathematical difficulties in the theoretical analysis of plastic deformation require applying a number of limitations. Plastic deformations in the solution under consideration are incomparably greater than elastic deformations; hence, the latter can be neglected. The materials used in friction surface production (aluminum, copper, zinc alloys, etc.) have a relatively low strain hardening, which makes it possible to consider them as hard ideally plastic materials subjected to plastic flow at almost constant shear stress $k$, slip-line fields are located at an angle of $45^{\circ}$ to the free surface. To analyze PDCS, the authors used an approximate energy method, which is based on extreme principles of the plasticity theory. The method supposes that the actual field of slip lines is replaced by a simpler kinematically admissible field, which consists of separate hard blocks and satisfies the boundary conditions in speeds and displacements. The power required for such deformation circuit exceeds the actual power of internal forces during plastic forming. Consequently, the specific efforts, which are computed using this method, are the top estimate of their actual values and allow establishing the maximum efforts for implementation of PDCS.

The kinematically admissible field of slip lines for plastic deformation during PDCS restoration is shown in Fig. 2. Blocks 4 and $4^{\prime}$ will be fixed referred to the adjoining surface 5 , and blocks 3 and $3^{/}$will move along the lines that are continuations of the boundaries of the indenter 1 and blocks 2 and $2^{\prime}$.

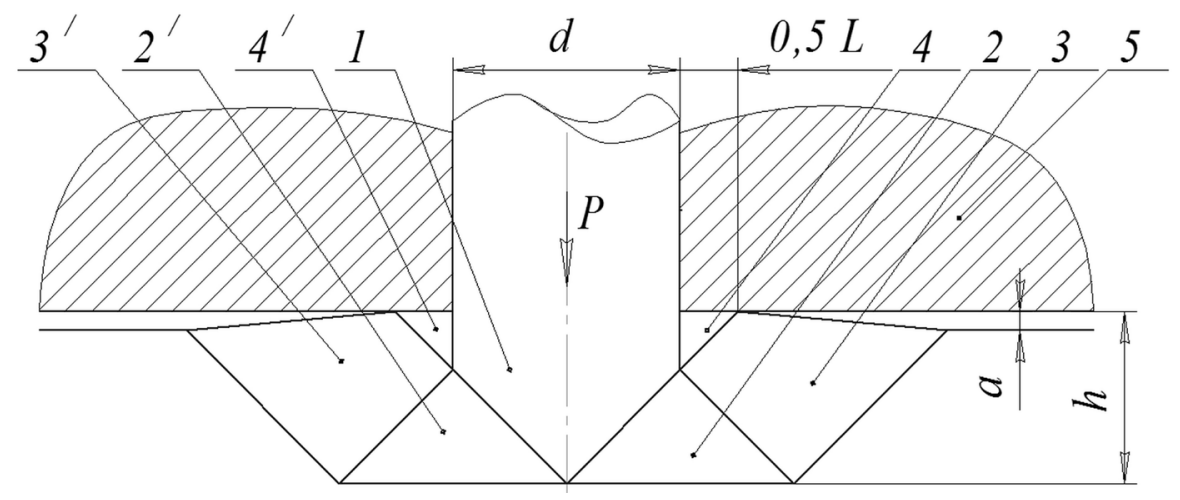

Fig. 2. The field of slip lines during worn surface restoration $(P$ is a penetration force, $d$ is an indenter diameter, $h$ is a penetration depth, $a$ is recovered wear, and $L$ is the length of the reconstructed surface).

\section{Results and Discussion}

Based on the energy method, we have obtained an equation for determining a contact pressure on an indenter working surface:

$$
q=4 k(1+\mu)(1+0.5 L / d),
$$

where $\mu$ is a friction coefficient between an indenter and a deformed material. 
A solution based on a plane problem is useless alone for a cone indenter, but many authors used the slip line method to solve an axisymmetric problem [11,12].

Based on the obtained equation, we determined a required penetration force and constructed a device for modeling PDCS at a local point (Fig. 3). This device allows measuring the depth of indenter penetration and determining the appropriate penetration force in the conditions of PDCS.

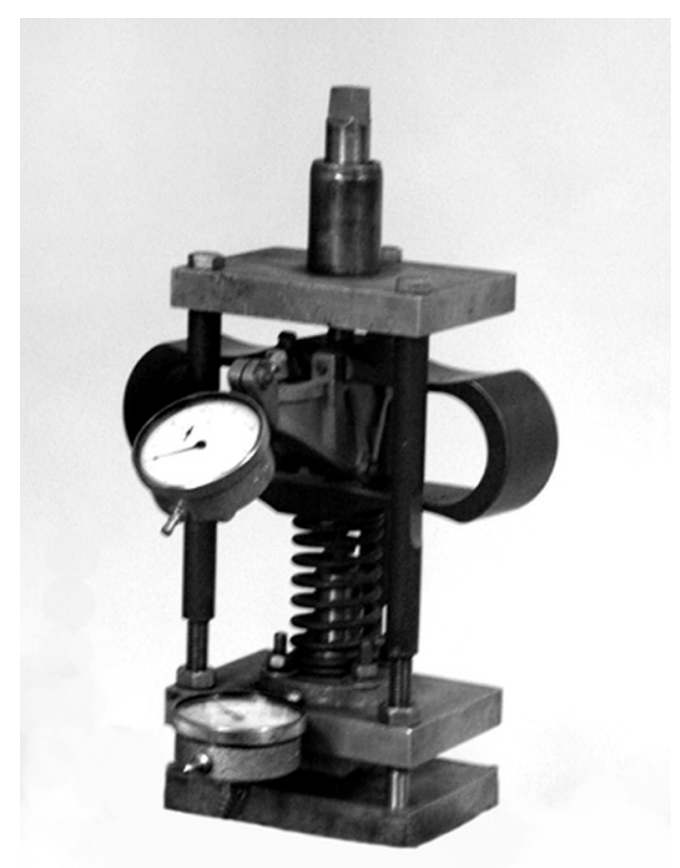

Fig. 3. A general view of a deformation tool.

To compare empirical and theoretical research, we used dimensionless values, which made it possible to compare the results in a more general form.

A dimensionless value $\Delta$ is obtained as the ratio of the force $P$ (required for indentor penetration to the depth $h$ ) to a particular force $P_{0}$. A dimensionless value $\delta$ is obtained as the ratio of the penetration depth $h$ to the indenter diameter $d$ (based on the experimental conditions $P_{0}$ was determined for the penetration depth $h=0.5 \mathrm{~mm}$, the indenter diameter $d$ from technological conditions was assumed $2 \mathrm{~mm}$ ). Since the value of the average contact pressure on the indenter working part varies depending on a penetration depth insignificantly (for indenters of the same shape), and a penetration force is proportional to a contact area, then the dependence of $\delta$ on $\Delta$ will not change when using indenters with other diameters.

Dependency graphs of the relative penetration $\delta$ on the relative force $\Delta$ obtained in tests on various materials together with theoretical graphs are shown in Fig. 4. During the restoration experiment using an adjoining conductor surface, we revealed the effect of increasing ductility. Thus, when studying cast iron, a sample was plastically deformed without fracture at the relative penetration $\delta=1.3$, although in similar tests without a conductor surface a control sample was destroyed already at the penetration of $\delta=0.5$. This indicates an increase in material ductility when using the proposed recovery method.

In Fig. 4 the interval $a$ is a deformation before touching the bounding surface by a deformed material (the penetration force increases due to an increasing contact area); the 
interval $b$ is a deformation after touching a bounding conductor by a restored surface (the curves deviate towards deformation simplification, which indicates a change in mechanical properties of materials); $c$ is a contact point of a deformed material and a conductor bounding surface.

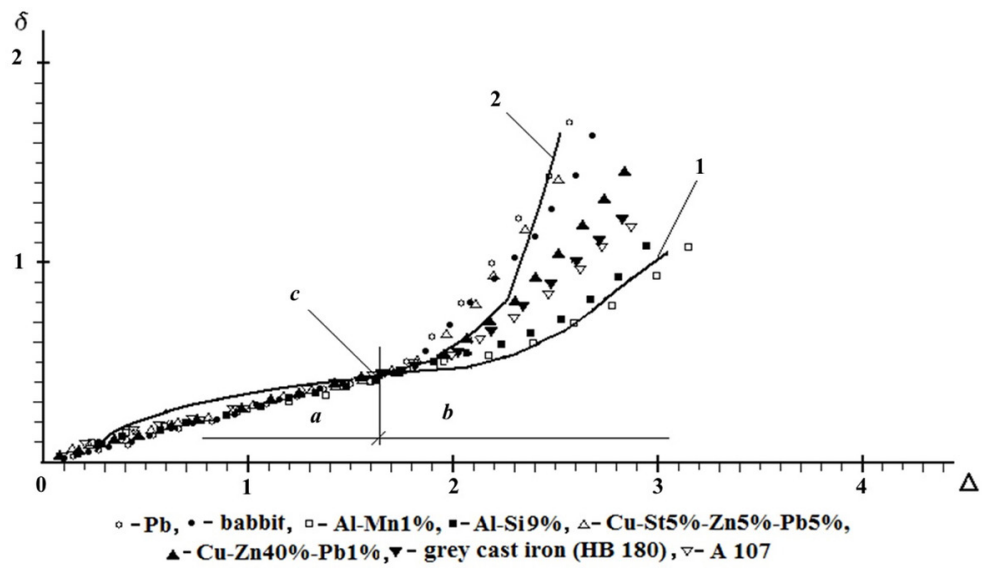

Fig. 4. The empirical and theoretical (a solid line) dependency graphs of the relative penetration $\delta$ on the relative effort $\Delta, 1$ is the curve plotted at the maximum friction coefficient of $0.5,2$ is at the friction coefficient of 0.14 .

Some discrepancy of curves in $b$ interval is due to different friction conditions of a deformed material along the cylindrical surface of the indenter. The graphs are arranged according to a friction coefficient of materials, the largest one belongs to aluminum alloys and the smallest one belongs to lead.

Thus, the theoretical solution is rather close to the results obtained in practice and reflects the basic regularities of the deformation process. The studied materials behave in a qualitative manner in the process of PDCS, regardless of their mechanical properties. After an adjoining conductor surface touches a deformed material, a pressure arises under the conductor, which creates a plastic core around the indenter. In this case, a pressure close to a hydrostatic pressure develops in the plastic zone (according to calculation results, the pressure in the plastic zone reached a value of about $6 k$ ), which changes mechanical properties of the material being deformed.

It should be emphasized that deformation with a boundary surface implements the effect of increasing ductility (even for relatively brittle materials such as cast iron). It allows significant deformations of the reconstructed surfaces without destruction. Moreover, due to deformation in a constraint environment, the effect of healing defects of a surface layer is achieved.

Other authors have also established the effect of increasing material ductility in their studies. Generally, ductility (deformation under destruction) increases with increasing hydrostatic pressure. In $[13,14]$, the properties of various materials were studied and the effect of pressure on ductility was considered in detail. It has been discovered that ductility increased with increasing pressure for all metals and alloys. When studying the deformation of cast iron, the microstructure of the longitudinal section of the sample before the test (Fig. 5, a) was compared to the deformed material of the sample neck tested at a pressure of $1750 \mathrm{MPa}$ to the stage immediately preceding the fracture (Fig. 5, b). 

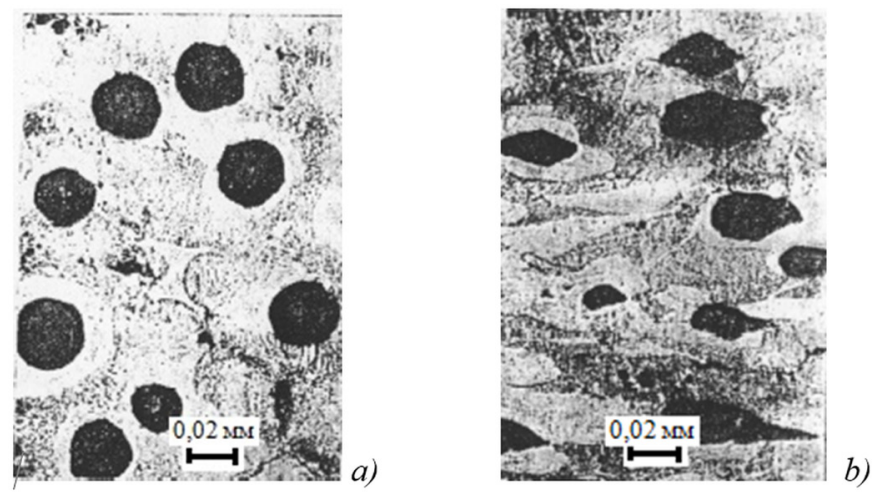

Fig. 5. Microstructure of cast iron tested for tension under pressure

Tensile testing of samples from spheroidal graphite cast iron under the pressure of 1750 $\mathrm{MPa}$ have shown that the true deformation at fracture, which is equal to zero at atmospheric pressure, increases with an increase in pressure to 1300-1400 $\mathrm{MPa}$. Because of various resistance to plastic deformation of ferrite and perlite sections that surround the spheroidal carbon spot, these carbon spot were stretched out.

\section{Conclusion}

The proposed recovery method can be used to repair peat cutter housing, couplings and other basic parts made of plastic materials. In addition, the developed method of plastic deformation restoration implements the effect of increased ductility of a material. The effect ensures a qualitative restoration of worn part surfaces made of materials with limited ductility, for example, from cast iron, which allows repairing bearing backs for rolling bearings of gearboxes and drive reducers of peat machines. Due to constrained deformation, the defects are healed in the surface layer, which positively affects wear resistance of a restored part [15]. There is an analytical expression that allows determining technological parameters of a recovery process for various materials.

\section{References}

1. Energy 2020. A strategy for competitive, sustainable and secure energy. Communication from the Commission to the European Parliament, the Council, the European Economic and Social Committee and the Committee of the Regions (ANCO, Brussels, 2010)

2. N. Kuznetsov, A. Mikhaylov, IPS, 1, 321328 (2000)

3. A. N. Bolotov, I. V. Gorlov, V. V. Novikov, ICIE, 2017, 606610 (2017)

4. H. P. Bloch, Major process equipment maintenance and repair (Gulf Publishing Company, Houston, Texas, 1998)

5. H. P. Bloch, Improving machinery reliability (Gulf Publishing Company, Houston, Texas, 1998)

6. V. Alexandrov, V. Gavdzinski, Thermal Stresses and Related Topics (LGFD, Rochester, 1997)

7. A. Smith, MSC Mentat Marc, A, 203220 (2012)

8. R. K. Pathria, Statistical mechanics (Elsevier, New York, 2011) 
9. F. J. Zerilli, R. W. Armstrong, J. Appl. Phys., 18161825, 88 (1987)

10. T. J. Holmquist, G. R. Johnson, J. J. de Phys. III, 853860 (1991)

11. R.T. Shield, Proc. Roy. Soc., 267, 89776 (1955)

12. F.J. Lockett, J. Mech. and Phys. of Solids, 345, 11988 (1963)

13. P. W. Bridgman, Studies in large plastic flow and fracture, 362 (McGraw-Hill, London, 1952)

14. B. I. Beresnev, Some problems of large plastic deformation of metals at high pressure, (Pergamon press, New York, 1963)

15. D.-H. Cho, S.-A. Lee, Y.-Z. Lee, Tribol. Lett, 45, 123129 (2012) 